\title{
Hierarchical synthesis based on Pareto-optimal fronts
}

\author{
E. Roca, R. Castro-López, F.V. Fernández \\ IMSE, CSIC and University of Sevilla \\ Sevilla, Spain \\ E-mail: Francisco.Fernandez@imse.cnm.es
}

\begin{abstract}
Pareto-optimal fronts have recently arisen as a promising alternative for design space exploration, potentially enabling better and more efficient hierarchical synthesis. This paper reviews the Pareto front generation problem, extends this concept to reconfigurable circuits, and discusses alternative applications to hierarchical synthesis approaches.
\end{abstract}

\section{INTRODUCTION}

Traditionally, most approaches to the automated synthesis of analog and mixed-signal circuits were focused on basic cells, with a few tens of devices. Although, initially, knowledge-based approaches became popular, most modern approaches are based on the iterative loop between an optimization loop and a performance evaluator, usually an electrical simulator.

However, designing more complex circuits requires decomposing the design problem into simpler problems by means of hierarchical decomposition. About 15 years ago, topdown hierarchical synthesis approaches were introduced, in which a circuit is designed with successively more detail [1]. Top-down design starts with the decomposition of the system specifications into a sub-set of specifications for each of the building blocks, commonly known as specification transmission. In doing so, the interactions between blocks are approximated to allow a certain independence of the design. The top-down process ends at the bottom level, where active and passive devices (transistors, resistors, and capacitors) compose the lowest level of abstraction of the blocks. For specification transmission at each hierarchical level, a similar method to that used at the cell level is applied: a performance evaluator embedded in an optimization loop. In most cases, an electrical simulator is not efficient enough, so high-level behavioral modeling and simulation techniques have to be applied (e.g., [2]).

The main advantage of the top-down design flow is that system performance is verified early in the process. However, a fundamental problem is that at intermediate hierarchical levels, there is no accurate information (only estimations, at best) on area occupation and power consumption [3]. The general criterion is to meet the high-level specifications while relaxing the specifications of the sub-blocks as much as possible, assuming that a sub-block with looser specifications can be designed with smaller area and power. But, if we take into account that, in general, some specifications of a sub- block can only be relaxed at the price of tightening others, there is no general criterion to decide which specifications or sub-blocks should be prioritized to get a better overall design. Moreover, specification transmission at the high level only guarantees, to a certain extent, that the high-level specifications can be met with the obtained sub-block specifications, but it is not at all guaranteed that those subblock specifications are realizable. These problems transform the top-down design process into an iterative process of unpredictable results and, eventually, high cost. Fortunately, this scenario may significantly change with the recent introduction of the so-called Pareto-optimal fronts (POF) for performance space exploration [4],[5].

Section II describes Pareto-optimal fronts and the computational techniques for obtaining them. Section III introduces useful extensions of POFs in order to use them for reconfigurable blocks, which are becoming increasingly important in analog and mixed-signal (AMS) design. Section IV is devoted to discuss two strategies for the application of POFs to hierarchical synthesis problems. Finally, conclusions are presented in Section V.

\section{PARETO Optimal Fronts}

The design goal is usually to minimize/maximize some objective function (e.g., minimize power consumption, minimize area), subject to some constraints (e.g., slew rate larger than a certain value), following the next formulation:

$$
\begin{aligned}
& \min _{\boldsymbol{x}} \boldsymbol{f}(\boldsymbol{x}) \\
& \text { subject to }\left\{\begin{array}{l}
\boldsymbol{g}(\boldsymbol{x}) \geq 0 \\
\boldsymbol{X}_{L}<\boldsymbol{x}<\boldsymbol{X}_{H}
\end{array}\right.
\end{aligned}
$$

where vector $\boldsymbol{y}=\boldsymbol{f}(\boldsymbol{x})=\left\{f_{1}(\boldsymbol{x}), f_{2}(\boldsymbol{x}), \cdots, f_{b}(\boldsymbol{x})\right\}$ represents the $b$ objective functions (i.e., performance characteristics) to be minimized/maximized, vector $\boldsymbol{x}$ corresponds to the design variables, and $\boldsymbol{X}_{L}$ and $\boldsymbol{X}_{H}$ are their lower and upper bounds, respectively. Vector $\boldsymbol{g}(\boldsymbol{x}) \geq 0$ corresponds to the user-defined constraints, delimiting the feasible region. A design point, $\boldsymbol{a} \in \boldsymbol{X}$, is said to dominate another design point, $\boldsymbol{b} \in \boldsymbol{X}, \quad$ (noted as $\quad \boldsymbol{a} \prec \boldsymbol{b}$ ) if $\quad \boldsymbol{F}(\boldsymbol{a}) \leq \boldsymbol{F}(\boldsymbol{b}) \quad$ and 
$f_{i}(\boldsymbol{a})<f_{i}(\boldsymbol{b})$ for at least one function $i^{1}$. The design point $\boldsymbol{a}$ is said to be non-dominated if there is no other design that dominates it. The non-dominated set of the entire feasible search space is known as the Pareto-optimal front (POF). The concepts of dominance and Pareto-optimal front are illustrated in Fig. 1 for a two-dimensional performance space.

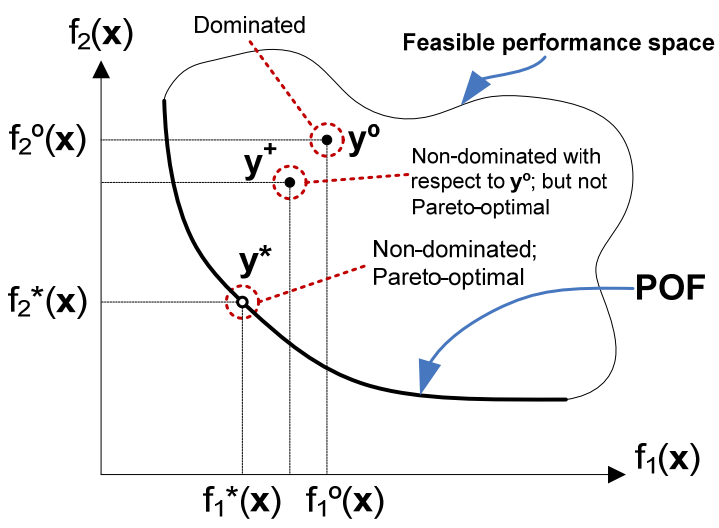

Fig. 1. Illustrating the Pareto-optimal front concept.

The efficient and accurate computation of the Pareto front constitutes a major challenge. Due to the practical impossibility to compute it analytically in most cases, the usual objective is to compute a set of samples of the POF. Regression techniques can be applied a posteriori to get the full set of trade-offs.

A first approach to solve this multi-objective optimization problem is to resort to multi-objective evolutionary algorithms [6]. Being of stochastic nature, the computational cost due to the high number of required fitness evaluations (circuit simulations) is the main drawback of these algorithms [4]. Efficiency, convergence to the true POF and diversity of solutions are areas of intense and current research. Efforts are done in this direction. An example is the definition of new quality evaluation metrics suitable to analog design problems proposed in [7]. These metrics are intended to enable performances comparisons of different multi-objective optimization algorithms or even comparison of sets of Pareto points from two independent runs of the same algorithm (e.g., with different algorithm parameter settings).

A second approach is to transform the multi-objective optimization problem into a sequence of single-objective optimization problems. This can be done, for instance, by applying a weighted sum of the objective functions or repetitively minimizing one objective function while constraints are imposed for the rest. Then, a single-objective optimization algorithm, usually a deterministic algorithm for efficiency reasons, is applied. An advanced implementation of this approach is reported in [5], that makes use of the normal boundary intersection method. The biggest drawback of these methods is that they might stuck at local minima due to the optimization algorithms applied. An improvement, reported in [8], is to try to escape from local minima thanks to the

\footnotetext{
This formulation is valid for minimization problems. A simple change of sign applies for maximization.
}

exchange of solutions with the other optimization algorithms executed in parallel.

Another possibility to avoid getting trapped in local minima is to apply a stochastic algorithm to each singleobjective optimization problem. However, this yields worse (less efficient or less accurate) results than an equivalent multi-objective evolutionary optimization problem with a population equal to the number of single-objective optimization problems.

\section{POFs OF RECONFIGURABLE CIRCUITS}

Reconfiguration in analog and RF circuits seeks to extend a circuit's functionality by combining circuit programming techniques and hardware sharing, so that the circuit can operate under many different targeted applications and varying external conditions without significantly increasing silicon area.

However, a regular POF cannot be easily used in the design of a reconfigurable system. Let us suppose a building block, whose POF is shown in Fig. 2, with two performance characteristics $f_{1}$ and $f_{2}$, that has to comply with specifications from two operation modes. During system-level design, we could pick one design for both modes (point 1) or different designs for each mode (points 2 and 3 ). Unfortunately, taking different designs implies that the circuit has to be reconfigured and, since points 2 and 3 can be completely different (in transistor sizes, in biasing conditions, etc.), it may render reconfiguration very difficult or even impossible to attain in practice. But if we take the same design (thus maximizing hardware sharing), there is the risk that not even a single design exists that simultaneously complies with specifications from both modes. Thus, system-level design becomes unrealizable.

To solve this, the multimode Pareto-optimal front concept has recently been introduced [9]. The concept itself is relatively simple, but significant changes in the dominance concept and the generation methodology have to be introduced.

A reconfigurable block addresses a group of $m \times b$ performance specifications, being $m$ the number of operation modes. Similarly, the vector of design variables must be extended by the number, $p$, of reconfigurability variables (i.e., the characteristics that change between circuit modes). To generate a multi-mode POF, for every visited point of the design space, $m$ performance evaluations are carried out to

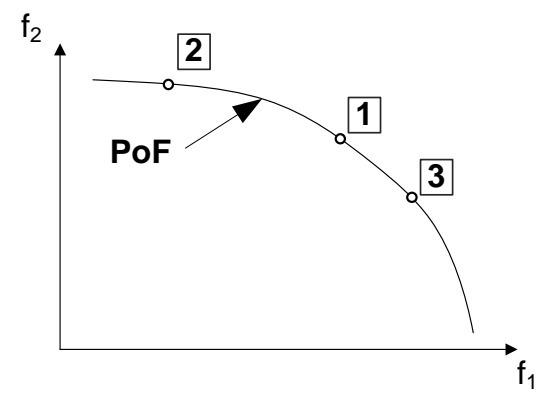

Fig. 2. Inability of regular POFs to handle multi-mode designs. 
evaluate the $b$ design objectives. In each evaluation, the $p$ reconfigurability variables are used to configure an operation mode. The outcome of this process is a $(m \times b)$ dimensional set which represents a $b$-dimensional POF of multiplicity $m$.

Pareto dominance sorting is required to set which individuals are best fitted, to guide the evolution-based optimization. In multi-mode dominance, the first issue to consider concerns the Pareto dominance between operation modes of the same individual: circuit performances on each separate operation mode must be non-dominated. This is illustrated in Fig. 3(a), showing an individual (in a minimization problem) with two modes represented by performances $\boldsymbol{y}^{*}$ and $\boldsymbol{y}^{\prime}$. Since $\boldsymbol{y}^{\prime}$ is dominated by $\boldsymbol{y}^{*}$, there is no use in taking this design into system-level design, since we could use instead $y^{*}$ in both operation modes. Therefore, an intra-individual sorting is first necessary, which compares all operation modes of the individual and removes those that are dominated. Performances $\boldsymbol{y}^{*}$ and $\boldsymbol{y}^{\prime \prime}$, are, on the other hand, non-dominated and, thus, can be used in system-level design.

A second issue pertains to the dominance between two individuals and their operation modes. Fig. 3(b) depicts two different solutions with 3 operation modes each. We can see that, though $\boldsymbol{y}_{1}^{i}$ and $\boldsymbol{y}_{3}^{i}$ dominate their counterparts $\boldsymbol{y}_{1}^{j}$ and $\boldsymbol{y}_{3}^{j}, \boldsymbol{y}_{2}^{i}$ is nonetheless non-dominated with respect to $\boldsymbol{y}_{2}^{j}$. If conventional dominance sorting were performed by considering the MM-POF as a $m \times b$ space, both solutions are not dominated. However, a designer would never use solution $j$ as long as solution $i$ is available. Therefore, an interindividual sorting is required, that compares the modes on an individual with all other individual's modes.

\section{POF-BASED HIERARCHICAL SYNTHESIS}

Different approaches to hierarchical synthesis based on the use of Pareto-optimal fronts can be considered. In this Section a previously reported bottom-up flow [10] is reviewed and a hybrid bottom-up/top-down flow is proposed. Advantages and limitations of both approaches are discussed.

\section{A. Bottom-up flow}

When designing a complex system, we would like to know its Pareto front. However, the complexity of the design space prevents such POF from being computed, even using

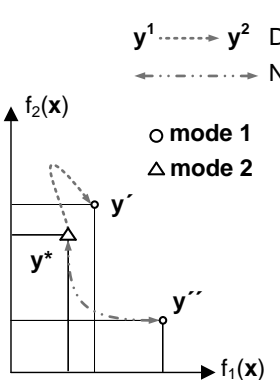

(a) hierarchical decomposition and behavioral simulation.

An approach recently proposed is to use Pareto fronts of lower-level sub-blocks to compose the Pareto front of the higher level block [10]. This idea is graphically illustrated in Fig. $4^{2}$. Therefore, starting from the POFs of the lower level sub-blocks, the hierarchy is traversed bottom-up until the POF of the complete system is generated. Although the computational cost of this approach can be extremely high, the advantages are obvious: once the high-level POF is generated, the complete set of trade-offs is available. Then, the designer can select a candidate (one sample of the POF) according to his/her preferences. As soon as it is selected, the full set of design variables at all hierarchical levels are determined.

However, as pointed out in [10], composition of fronts cannot be performed carelessly. The idea described above can only be exploited under the assumption that we only need the lower level POFs to compose the higher level POFs. This implies that no point in the true POF of a block can originate from a dominated point of a lower level sub-block. Consider, for the sake of simplicity, two performance objectives, $f_{1}^{i}$ and $f_{2}^{i}$,of a block at level $i$, that depend of two objectives of a sub-block at level $i-1$. This is mathematically stated as [10]:

$$
\frac{\delta f_{1}^{i}}{\delta f_{1}^{i-1}}>0 \quad \frac{\delta f_{1}^{i}}{\delta f_{2}^{i-1}}>0 \quad \frac{\delta f_{2}^{i}}{\delta f_{1}^{i-1}}>0 \quad \frac{\delta f_{2}^{i}}{\delta f_{2}^{i-1}}>0
$$

with only the following possible exceptions:

$$
\begin{aligned}
& \text { if } \frac{\delta f_{1}^{i}}{\delta f_{1}^{i-1}}<0 \text { and } \frac{\delta f_{1}^{i}}{\delta f_{2}^{i-1}}>0 \text { then }\left\|\frac{\delta f_{1}^{i}}{\delta f_{1}^{i-1}}\right\|<\left\|\frac{\delta f_{1}^{i}}{\delta f_{2}^{i-1}}\right\| \\
& \text { if } \frac{\delta f_{1}^{i}}{\delta f_{1}^{i-1}}>0 \text { and } \frac{\delta f_{1}^{i}}{\delta f_{2}^{i-1}}<0 \text { then }\left\|\frac{\delta f_{1}^{i}}{\delta f_{1}^{i-1}}\right\|>\left\|\frac{\delta f_{1}^{i}}{\delta f_{2}^{i-1}}\right\| \\
& \text { if } \frac{\delta f_{2}^{i}}{\delta f_{1}^{i-1}}<0 \text { and } \frac{\delta f_{2}^{i}}{\delta f_{2}^{i-1}}>0 \text { then }\left\|\frac{\delta f_{2}^{i}}{\delta f_{1}^{i-1}}\right\|<\left\|\frac{\delta f_{2}^{i}}{\delta f_{2}^{i-1}}\right\| \\
& \text { if } \frac{\delta f_{2}^{i}}{\delta f_{1}^{i-1}}>0 \text { and } \frac{\delta f_{2}^{i}}{\delta f_{2}^{i-1}}<0 \text { then }\left\|\frac{\delta f_{2}^{i}}{\delta f_{1}^{i-1}}\right\|>\left\|\frac{\delta f_{2}^{i}}{\delta f_{2}^{i-1}}\right\|
\end{aligned}
$$

These conditions must hold in all points of the feasible search space. In other words, they state that the movement of a solution at level $i-1$ towards the Pareto front must yield a movement of the corresponding solution towards the Pareto front at level $i$. These conditions must be studied for each block and each set of performances. This study may determine which performances can be used as objective functions, and even if this approach can be applied to a given system.

\section{B. Hybrid bottom-up top-down flow}

As discussed above, the bottom-up flow introduced above has numerous advantages. However, we have to consider that the generation algorithms are only able to produce a limited number of points of each POF. This means that when the POFs are composed up the hierarchy like in Fig. 4, only a

\footnotetext{
${ }^{2}$ For illustration's sake, the POFs of the different blocks have been drawn for performances that either have to be minimized or maximized.
} 


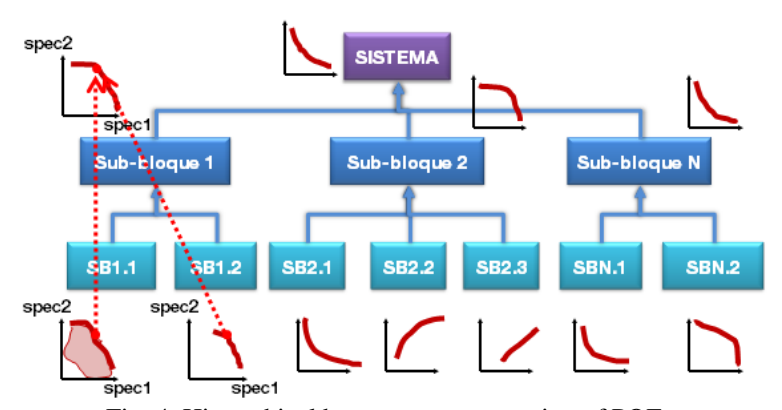

Fig. 4. Hierarchical bottom-up construction of POFs.

finite number of samples of the POF of the system is available. Consider for instance that the two-dimensional POF of the system in Fig. 4 represents signal-to-noise ratio versus power consumption. If we desire a given SNR, it is obvious that we will select the point with minimum power and SNR larger than the desired one. If we do not want to waste power, it is desirable to have a sample close to the desired SNR value. This requires that the sampling of the POF should be sufficiently dense. If we consider that in general that each block at each hierarchical level can have a relatively large number of performances of interest, a dense sampling of the POFs may be computationally very expensive.

In this situation, a new possibility arises. Given a set of samples, regression techniques can be used to obtain a performance model. This performance model can be used to generate POFs at higher hierarchical levels and apply a topdown optimization process. In this way the drawback of the bottom-up flow is avoided. The drawbacks of the conventional top-down design approach are also avoided:

- Optimization at each hierarchical level is performed knowing the best trade-offs (not just samples) offered by lower level sub-blocks.

- Optimization at each hierarchical level is performed knowing that the obtained performances of lower level sub-blocks are realizable.

- Optimization at each hierarchical level is performed with information on characteristics of lower level subblocks like power and area, which are essential for appropriate balance of specifications in the top-down process.

The potential capability of this approach to produce a better solution than the bottom-up flow and the conventional top-down design approach is obvious. It may be argued that the price to pay is a much higher computational cost. However, two aspects have to be considered:

- As stated above, the limitations of the top-down design approach transform it in an iterative process. Such iterations are largely, if not completely, eliminated.
- $\quad$ The computational cost of POF generation grows with the number of samples (related to the population size in evolutionary algorithms). If the samples are going to be used in the previous bottom-up flow it is expected that their number should be much higher than if they are going to be used just to build performance models by means of regression techniques.

A further exploration of the best trade-offs is an open research point.

\section{CONCLUSION}

The potential of Pareto-based analog synthesis is evident, despite the problems yet to be solved. This paper has discussed some major advances on POF generation and its application techniques. As this paradigm matures, there is no doubt that increasing interest will be attracted in coming years.

\section{REFERENCES}

[1] H. Chang, E. Charbon, U. Choudhury, A. Demir, E. Felt, E. Liu, E.Malavasi, A. Sangiovanni-Vincentelli and I. Vassiliou, A Top-Down, Constraint- Driven Design Methodology for Analog Integrated Circuits, Springer, 1996.

[2] J. Ruiz-Amaya, José M. de la Rosa, Francisco V. Fernández, Fernando Medeiro, R. del Río, B. Pérez-Verdú and A. Rodríguez-Vázquez, "High-Level Synthesis of Switched-Capacitor,Switched-Current and Continuous-Time $\Sigma \Delta$ Modulators Using SIMULINK-Based TimeDomain Behavioral Models, IEEE Trans. Circuits and Systems-I, Vol. 52, No. 9, pp. 1795-1810, Sept. 2005.

[3] E. Lauwers and G. Gielen, "Power Estimation Methods for Analog Circuits for Architectural Exploration of Integrated Systems,” IEEE Trans. On VLSI, vol. 10, No. 2, pp. 155-162, April 2002.

[4] B. de Smedt and G. Gielen, "WATSON: Design Space Boundary Exploration and Model Generation for Analog and RF IC Design," IEEE Trans. on Computer-Aided Design, Vo. 22, No. 2, pp. 213-224, Feb. 2003.

[5] G. Stehr, H. Graeb and K. Antreich, “Analog Performance Space Exploration by Normal-Boundary Intersection and by Fourier-Motzkin Elimination,” IEEE Trans. on Computer-Aided Design, Vol. 26, No. 10, pp. 1733-1748, Oct. 2007.

[6] K. Deb, Multi-objective Optimization Using Evolutionary Algorithms. Wiley, 2001.

[7] F.V. Fernández, B. Liu, R. Castro-López, E. Roca, "Quality Metrics of Pareto-optimal Fronts for Multi-objective Synthesis of Analog IC," Proc. of the Int. Workshop on Symbolic and Numerical Methods,Modeling and Applications to Circuit Design, pp.56-60, 2008.

[8] D. Mueller, H. Graeb and U. Schlichtmann, "Trade-off Design of Analog Circuits Using Goal Attainment and Wave Front Sequential Quadratic Programming,” Proc. Design, Automation and Test in Europe Conf., 2007.

[9] R. Castro-López, E. Roca and F.V. Fernández, "Multimode Pareto Fronts for Reconfigurable Analogue Circuits,” IET Electronics Letters, Vol. 45, No. 2, Jan. 2009.

[10] T. Eeckelaert, T. McConaghy and G. Gielen, "Efficient Multiobjective Synthesis of Analog Circuits Using Hierarchical Pareto-optimal Performance Hypersurfaces,” Proc. Design, Automation and Test in Europe Conf., 2005. 